\title{
Génétique moléculaire des maladies kystiques rénales
}

En 1985, le gène principal (PKI) 1 ) de la polykystose rénale autosomique dominante (ADPKD) était localisé au bras court du chromosome 16 . Les néphrologues découvraient la génétique moléculaire et les généticiens se rappelaient qu'il existait des maladies rénales héréditaires dont l'ADPKD, une des maladies héréditaires monogéniques les plus fréquentes dans l'espèce humaine.

1994 est une "grande année " pour la génétique des maladies kystiques rénales héréditaires. En effet, le gène $P K I) 1$ a été identifié par le European Polycystic Kidney Disease Consortium, comme l'a indiqué un flash dans médecine/sciences ( $n^{\circ}$ 6-7, vol. 10 , p. 760). Ce résultat a été obtenu en partie grâce à l'étude d'une famille portugaise dans laquelle coexistaient ADPKD et sclérose tubéreuse de Bourneville dont un gène (TSC2) a été localisé au bras court du chromosome 16, à proximité de PKD1 (voir $m / s n^{\circ} 5$, vol. 10, p. 601). Dans cette famille, la mère et la fille sont atteintes d'ADPKD et ont une translocation équilibrée entre le bras court du chromosome 16 et le chromosome 22 . Le point de cassure touche le gène $P K D$ l. Dans la même famille, le fils est atteint de sclérose tubéreuse et a une monosomie pour l'extrémité du bras court du chromosome 16 puisqu'il n'a pas hérité de la translocation équilibrée. Cela démontre que le gène TSC2 est délété et est situé en position distale par rapport au point de cassure [1]. Depuis plusieurs années, on savait qu'il existait des familles non PKD 1 . En 1993, un second locus a été localisé, $P K D 2$ ou 4, au bras court du chromosome 4 par deux équipes qu'il existe encore un autre locus. Les formes non $P K I) 1$ représentent environ 10 à $15 \%$ des cas; l'évolution des kystes et de la maladie semble plus lente que dans les formes $P K D 1$.

Le gène de la polykystose rénale autosomique récessive (ARPKD) vient d'être localisé à la région chromosomique 6p21-cen par Zerres et al. réunissant des groupes allemands, français, finlandais et belges [4]. La maladie ARPKD se révèle dans l'enfance : les kystes rénaux dérivent des canaux collecteurs (alors qu'ils dérivent de tous les segments tubulaires dans ADPKD); une atteinte hépatique, dénommée "fibrose hépatique congénitale ", est associée comportant une dysgénésie biliaire et une fibrose portale responsable d'une hypertension portale avec hémorragie digestive. L'incidence de l'ARPKD est d'environ 1/40 000 à la naissance. La localisation du gène devrait faciliter le diagnostic prénatal dans les familles atteintes.

De multiples modèles expérimentaux de polykystose rénale ont été étudiés, certains ressemblant à ARPKD, y compris une souche de souris transgéniques $\mathrm{TgN} 737 \mathrm{Rpw}$ établie par Moyer et al. [5]. Le gène en cause a été localisé chez la souris sur le chromosome 14 (dont le locus humain correspondant se situe sur le chromosome 13). Ce dernier locus a été exclu par Zerres et al. dans ARPKD [4]. Dans deux autres souches de souris avec kystes rénaux et hérédité récessive autosomique, $p c y$ et $c p k$, les gènes murins, ont été localisés respectivement aux chromosomes 9 et 12, sans concourir à la localisation du gène chez l'homme.
Le gène de la néphronophthise juvénile, appelée par certains "maladie kystique de la médullaire rénale ", a été localisé en 1993 par Antignac et al. [6] au bras court du chromosome 2. Dans certaines familles, la néphronophthise est associée à une dégénérescence tapéto-rétinienne (syndrome de Senior-Loken); ces cas ne sont pas liés au locus situé en $2 p$. Il existe donc une hétérogénéité génétique; dans ARPKD, Zerres et al. n'ont pas mis en évidence d'hétérogénéité génétique malgré la nonuniformité de la présentation clinique.

Des kystes rénaux sont observés enfin dans certaines phacomatoses, la sclérose tubéreuse et la maladie de von Hippel-Lindau pour lesquelles des informations décisives de génétique moléculaire ont été acquises récemment. Deux loci ont été identifiés pour la sclérose tubéreuse, TSC1 au bras long du chromosome 9 et TSC 2 au bras court du chromosome 16 (voir plus haut). Des kystes rénaux multiples peuvent être observés dans les deux formes [1]. Dans l'étude publiée en 1994, les auteurs signalent un cas où existe une délétion d'environ $100 \mathrm{~kb}$, touchant à la fois TSC2 et PKI)1. Le malade a une sclérose tubéreuse et une maladie polykystique rénale particulièrement sévère [1]. Dans la maladie de von Hippel-Lindau, les kystes rénaux sont associés habituellement à des carcinomes rénaux, souvent bilatéraux et multifocaux. Le gène $V H L$ a été identifié en 1993 (voir $m / s n^{\circ} 8-9$, vol. 9, p. 988).

Et maintenant? Tout, ou presque tout, reste à faire : quels sont les mécanismes qui conduisent à la formation des kystes rénaux et com- 
ment s'y opposer? Nous n'avons pas d'information sur les produits des gènes concernés ; il est très probable que la kystogenèse dépende de plusieurs mécanismes moléculaires, ce qui, a priori, n'était pas évident pour une structure apparemment simple, un kyste, c'est-à-dire une cavité contenant un liquide entourée d'un épithélium. Pourquoi les kystes sontils limités au rein dans beaucoup de formes, ou associés très souvent à des kystes hépatiques dans ADPKD ou à une fibrose hépatique dans ARPKD ? Beaucoup de questions posées, mais avec l'espoir d'y répondre car les outils nécessaires à la compréhension ont progressé en 1993-1994.

J.P.G.

1. The European Polycystic Kidney Disease Consortium. The polycystic kidney disease 1 gene encodes a $14 \mathrm{~kb}$ transcript and lies within a duplicated region on chromosome 16. Cell 1994 ; $77: 88194$.

2. Peters I)JM, Spruit I, Saris IJ, Ravine D, Sandkuijl I.A, Fossdal R, Boersma J, van Eijk R, Norby S, Constantinou-Deltas C.D, Pierides A. Brissenden JE, Frants RR, van Ommen GJB, Breuning $\mathrm{MH}$. Chromosome 4 localization of a second gene for autosomal dominant polycystic kidney disease. Nature (jenet $1993 ; 5: 359-62$.

3. Kimberling W'J, Kumar S, Gabow PA, Kenyon JB, Connolly C.J, Somlo S. Autosomal dominant polycystic kidney disease : localization of the second gene to chromosome 4q13-q23. Cienomics $1993 ; 18: 467-72$

4. Zerres K, Mucher G, Bachner I, Des chennes $\mathrm{G}$, Eggermann $\mathrm{T}$, Kääriäinen $\mathrm{H}$, Knapp M, L.ennert T, Misselwitz J, von Mühlendahl KE, Neumann HPH, Pirson Y, Rudnik-Schöneborn S, Steinbicker V', W'irth B, Schärer K. Mapping of the gene for autosomal recessive polycystic kid ney disease (ARPKI) to chromosome 6p2l-cen. Nature Genet 1994; $7: 429-32$.

5. Moyer JH, I,ee-Tischler MJ, Kiwon $\mathrm{HJ}$ Schrick IJ, Avner EI), Sweeney W'E, Godfrey VI. Cacheiro NIA, Wilkinson JE, W'oychik RP. Candidate gene associated with a mutation causing recessive polycystic kidney disease in mice. Science $1994 ; 264: 1329-33$

6. Antignac C, Arduy CH, Beckmann JS, Benessy F, Gros F, Medhioub M, Hildebrandt F, Dufier JI, Kleinknecht C, Broyer M, W'eissenbach J, Habib R, Cohen I). A gene for familial juvenile nephronophthisis (recessive medullary cystic kidney disease) maps to chromosome 2p. Nature Cienet $1993 ; 3: 342-5$

$\mathrm{m} / \mathrm{s} n^{\circ} 10$, vol. 10, octobre 94
Activité tyrosine kinase du récepteur de l'insuline et translocation de GLUT4. L'insuline stimule le transport du glucose dans l'adipocyte par translocation du transporteur GLUT4 vers la membrane plasmique. L'implication de l'activité tyrosine kinase du récepteur dans cet effet aigu de l'hormone demeure l'objet de vives controverses. Cette question a été réévaluée par l'examen des conséquences fonctionnelles d'une mutation du récepteur de l'insuline $\left(\mathrm{Met}^{1153} \rightarrow\right.$ Ile) affectant son activité tyrosine kinase, trouvée chez un patient insulinorésistant [1]. L'originalité de cette étude repose sur l'utilisation d'adipocytes transfectés transitoirement, système cellulaire remarquablement sensible à l'insuline. Des adipocytes de rat ont été cotransfectés avec l'ADNc du récepteur de l'insuline muté ou normal et l'ADNc de GLUT4 comportant un épitope hémagglutinine (HA) (12 acides aminés d'une hémagglutinine) dans un domaine extracellulaire. A l'aide d'anticorps anti-HA, on peut mesurer l'apparition de GLUT4 sur la membrane plasmique en réponse à l'insuline spécifiquement sur les cellules transfectées. Alors que la surexpression du récepteur normal augmente le recrutement de GLUT4-HA, la surexpression du récepteur muté n'a pas d'effet : elle conduit à une réponse à l'insuline identique à celle observée dans les cellules témoins cotransfectées avec le vecteur vide. Le récepteur muté ne transmet donc pas le signal nécessaire à la translocation de GLUT4 et n'exerce pas d'effet négatif dominant sur le récepteur endogène. Ces résultats militent en faveur d'un rôle essentiel de l'activité tyrosine kinase du récepteur de l'insuline dans la stimulation du transport du glucose. Ce système devrait permettre de décrypter la voie de signalisation de cet effet majeur de l'insuline, directement sur une cellule cible.

[1. Quon MJ, et al. Proc Natl Acad Sci USA 1994 ; 91 : 5587-91.]
Le CNTF joue-t-il un rôle physiologique? La question mérite d'être posée au regard des résultats présentés par Ryosuke Takahashi $e t$ al. (Metropolitan Institute for Neuroscience, Tokyo, Japon). Ces auteurs ont en effet obtenu fortuitement deux clones d'ADNc en criblant une banque construite à partir de nerfs sciatiques humains à l'aide d'une sonde composée d'un fragment du gène codant pour le CNTF [1]. L'un des ADNc comprenait un cadre de lecture ouvert pour 200 acides aminés identique à celui prédit par l'ADN génomique du CNTF. L'autre, en revanche, contenait une insertion de quatre paires de base provoquant un décalage du cadre de lecture au niveau du trente-neuvième acide aminé et un signal stop après formation d'une protéine de 62 acides aminés. Or l'analyse de deux larges populations d'individus sains (cent cinquante) et de patients atteints de maladies neurologiques diverses (deux cent quarante) a révélé, parmi les premiers, cinquante-deux hétérozygotes et quatre homozygotes pour la mutation et, parmi les seconds, cinq homozygotes sans qu'aucun lien puisse être fait avec une affection particulière. Il semble donc, globalement, que l'invalidation du gène du CNTF chez l'homme ne soit pas associée à une anomalie majeure. Ces résultats restent un peu troublants, car une souris au gène CNTF invalidé a, à l'inverse, présenté au long cours une atteinte motoneuronale, même si celle-ci est restée très partielle [2]. Troublant aussi lorsque l'on sait que le gène codant pour le CNTF est très conservé, ce qui plaiderait plutôt en faveur d'un rôle essentiel.

[1. Takahashi R, et al. Nature Genet $1994 ; 7$ : 79-84.]

[2. Masu Y, et al. Nature 1993 ; 365 : 27-32.] 
Le récepteur $\mathrm{AT}_{2}$ de l'angiotensine II : un membre atypique de la superfamille des récepteurs à sept segments transmembranaires. L'hormone angiotensine II (AII) exerce ses effets physiologiques en interagissant avec deux sous-types de récepteurs membranaires, identifiés $\mathrm{AT}_{1}$ et $\mathrm{AT}_{2}$. Les études pharmacologiques ont montré que le récepteur $\mathrm{AT}$ est exprimé dans la grande majorité des tissus cibles de l'AII et est responsable des principaux effets connus de l'hormone [ 1 , 2]. Le récepteur $\mathrm{AT}_{\text {, }}$ est caractérisé par sa forte affinité pour l'antagoniste non peptidique losartan (Dup 753), de même que par sa sensibilité aux nucléotides guanyliques et aux agents réducteurs. Au niveau moléculaire, le récepteur AT, possède une structure à sept segments transmembranaires, caractéristique des récepteurs couplés aux protéines G. L'activation du récepteur $\mathrm{AT}_{1}$ est couplée à de multiples voies de signalisation, incluant la stimulation de la phospholipase C et l'inhibition de l'adénylyl cyclase. Au contraire, on connaît peu de choses sur les mécanismes de signalisation et sur les rôles physiologiques du sous-type $\mathrm{AT}_{2}$. Le récepteur $\mathrm{AT}_{2}$ est caractérisé pharmacologiquement par sa forte affinité pour l'antagoniste PD 123319 et sa faible affinité pour le losartan, de même que par son insensibilité aux nucléotides guanyliques. Le clonage récent de l'ADNc du récepteur $\mathrm{AT}_{2}$, par les équipes de Dzau (Stanford, USA) et Inagami (V'anderbilt, USA) devrait aider à élucider la fonction de ce récepteur [3, 4]. Les deux équipes ont utilisé une approche de clonage par expression pour isoler l'ADNc du récepteur. L'analyse de la séquence d'acides aminés révèle que le récepteur $\mathrm{AT}_{\text {, }}$ ne possède qu'une analogie de $32 \%$ avec le sous-type $\mathrm{AT}_{1}$. Fait intéressant, le récepteur $\mathrm{AT}_{\text {, }}$ montre également une structure à sept segments transmembranaires potentiels, en dépit de l'absence de sensibilité de la liaison des agonistes aux nucléotides guanyliques. Par ailleurs, la comparaison de la séquence du récepteur $\mathrm{AT}_{\text {, à }}$ d'autres récepteurs de cette famille suggère l'existence d'un motif de cinq acides aminés qui serait présent dans la troisième boucle cytoplasmique d'un certain nombre de récepteurs dont les propriétés de liaison sont insensibles au GTP. En plus du récepteur $\mathrm{AT}_{\text {, }}$, ce motif est également retrouvé dans le récepteur SSTRl de la somatostatine et le récepteur $\mathrm{D}_{3}$ dopaminergique. Ces observations suggèrent au groupe de Dzau que ce motif pourrait définir une classe atypique de récepteurs avec une topologie à sept segments transmembranaires mais dépourvus de couplage aux protéines $G$ classiques [3]. Il est intéressant de noter que les récepteurs $\mathrm{AT}_{2}, \mathrm{SSTRl}$ et $\mathrm{D}_{2}$, de même qu'un récepteur du VIP insensible au GTP, sont fortement exprimés dans les tissus fotaux et neuronaux. Ces récepteurs pourraient être couplés à de nouveaux mécanismes de signalisation et jouer un rôle critique dans le développement embryonnaire.

[1. Timmermans P, et al. Pharnacol Rev 1993 ; 45 : 205-51.]

[2. Corvol $\mathrm{P}$, et al. médecine/sciences $1993 ; 9$ : 1050-60.]

[3. Mukoyama M, et al. J Biol Chem 1993 ; 268 : 24539-42.]

[4. Kambayashi Y, et al. J Biol Chem 1993 ; 268 : 24543-6.]

JAK3, une nouvelle Janus kinase, relais de transmission $\mathrm{du}$ signal d'IL2 et IL4. La liaison d'IL2 à son récepteur des cellules $\mathrm{T}$ cytotoxiques entraîne la phosphorylation du récepteur par des kinases de la famille src, en particulier Lck. Cependant, la signalisation d'IL2 peut être transmise en l'absence de Lck, ce qui a fait rechercher une nouvelle tyrosine kinase. Une kinase de la famille Janus, JAK3 vient d'être découverte de façon concomitante par deux équipes américaines, celle de Ihle à Memphis (TE USA) et celle de O'Shea au National Cancer Institute à Frederick (MD USA) [1, 2]. Elle est le relais de transmission du signal dans les cellules lymphoïdes et myéloïdes en réponse à IL2 et IL 4. De masse molaire $120 \mathrm{kDa}$, sa séquence en acides aminés est respectivement $47 \%$ et $36 \%$ similaire à celles de JAKl et JAK2. Elle contient au moins un domaine catalytique tyrosine kinase. Contrairement aux autres membres de la famille, JAKl et JAK2, qui sont ubiquitaires $(\mathrm{m} / \mathrm{s}$ $n^{\circ} 2$, vol. 10, p. 202, [3]), l'expression de JAK3 est restreinte aux tissus myéloïdes et lymphoïdes. Son activation est très spécifique du signal relayé par IL2 et IL4. Elle n'est pas activée par la liaison à leurs récepteurs de l'érythropoïétine, de IL3, de GM-CSF, des interférons $\alpha$ et $\gamma$ ni de IL6, dont, pourtant, les domaines cytoplasmiques sont très semblables à celui de la chaîne $\beta$ du récepteur de l'IL2. Celui-ci comporte un domaine juxtamembranaire riche en sérine, indispensable à l'activation de JAK3 et un domaine acide indispensable à l'activation de $\mathrm{p} 56^{l \mathrm{l} k}$, p59/yn ou p53/56 $6^{\operatorname{lyn} 18}$ qui n'est pas impliqué dans la division cellulaire. IL2 et IL4 activent toujours JAK3 dans les cellules myéloïdes et activent aussi JAKl dans les C.TL. L'activation concomitante de JAKl et de JAK3 est-elle nécessaire à la réponse des lymphocytes $\mathrm{T}$ ?

[1. Johnston JA, et al. Nature 1994 ; 370: 151-3.]

[2. Witthuhn BA, et al. Nature 1994 ; $370: 153-7$.

[3. Dusanter I, et al. médecine/sciences $1994 ; 10: 825-35$.]

La " barrière hémato-encéphalique " n'est pas une barrière, c'est un filtre ! S'il existe un terme malheureux, c'est bien celui de barrière hémato-encéphalique, en ce qu’il évoque à tort l'existence d'un obstacle mécanique au passage des substances et éléments circulants vers le cerveau. Or il n'existe rien de tel et, sans parler des cellules qui traversent sans gros problème apparent la paroi vasculaire par diapédèse, l'obstacle mis au passage des molécules est très différent de l'une à l'autre selon des critères biologiques complexes parmi lesquels la taille elle-même n'est qu'un 
des déterminants. Ce filtre biologique différentiel vient d'être réétudié en parallèle pour cinq macromolécules, l'insuline, le NGF, l'albumine, la transferrine et des IgG chez le rat, en calculant la diffusion intraparenchymateuse après injection intraveineuse ou in traartérielle en bolus [1]. Les résultats démontrent la complexité du filtre puisque les IgG et l'albumine ne passent que très faiblement, alors que le NGF et la transferrine passent 15 à 60 ) fois mieux et l'insuline 300 fois ! Par ailleurs, il existe des différences régionales notables et le NGF qui passe un peu moins bien que la transferrine dans le thalamus ou le tronc cérébral est, en revanche, mieux capté au niveau d'un de ses sites d'action habituels, l'hippocampe. Ces résultats ne sont pas vraiment inattendus, car les valeurs pour chacune des molécules avaient déjà été obtenues indépendamment. l.eur comparaison systématique souligne toutefois les caractéristiques différentielles de la prétendue barrière, ce qui méritait d'être refait.

[1. Poduslo JF, et al. Proc Natl Acad Sci LSA 1994; 91 : 5705-9.]

Inodisomie du chromosome 6 avec acidémie méthylmalonique et diabète. Une équipe belge [1] relate l'histoire d'un nouveau-né décédé à 16 jours dans un tableau d'acidose. Un diagnostic d'acidémie méthylmalonique avait pu être posé. On sait qu'il s'agit d'une maladie autosomique récessive, dont le locus se trouve en 6p21, lié au complexe majeur d'histocompatibilité (CMH) et proche du locus de l'ataxie spinocérébelleuse SCA1. Ce locus appelé MUT (méthylmalonyl CoA mutase) possède un déficit connu sous le nom de mut ${ }^{0}$. A l'analyse anatomique, on constatait la présence d'un pancréas et d'îlots apparemment normaux, mais aucune cellule ne produisait d'insuline; on n'observait pas d'anticorps antiîlots ou anti-insuline. Une particularité importante de l'ADN de cet enfant était l'absence de marqueurs du chromosome 6 provenant de la mère, absence s'étendant probablement sur toute la longueur du chromosome, en tout cas au moins sur $25 \mathrm{cM}$, incluant le CMH et SCA I. Il s'agissait donc d'une disomie uniparentale; de plus, les marqueurs étant homozygotes, on était en présence d'une isodisomie, c'est-à-dire que les deux chromosomes $6 \mathrm{du}$ malade provenaient d'un même chromosome 6 paternel dupliqué $\left(\mathrm{m} / \mathrm{s} n^{\circ} 1\right.$, vol. 6, p. 57). Des deux symptômes ayant affecté cet enfant, acidémie méthylmalonique et diabète insulinodépendant, le premier s'explique aisément. Seul le père était porteur du trait, à l'état hétérozygote ; l'absence de contribution maternelle et la duplication du chromosome atteint l'ont conduit à l'état homozygote. En revanche, aucun locus sur le 6 n'est connu pour commander la production de cellules $\beta$ ou d'insuline. En ne tenant pas compte de la possibilité d'une coïncidence fortuite, plusieurs hypothèses restent possibles : (1) l'existence d'un gène de développement sur le chromosome 6 , impliqué dans la différenciation des cellules $\beta$. On peut même imaginer qu'une microdélétion, invisible au microscope, puisse affecter à la fois le gène $M U T$ et celui des cellules $\beta$. Cette hypothèse, plausible, n'a actuellement aucun appui expérimental; (2) une activation des gènes HLA pourrait aboutir à une agénésie des cellules $\beta$, idée fondée sur des expériences de transgenèse effectuées avec des antigènes de classe I ou II [2] ; (3) une "empreinte génomique" du chromosome 6 pourrait rendre inactif le locus des cellules $\beta$ si les allèles maternels sont seuls exprimés. Contre les deux dernières hypothèses milite une observation connue d'isodisomie du 6 ([3] et $\mathrm{m} / \mathrm{s} n^{\circ} 8$, vol. $6, p .827$ ) dans laquelle la totalité du 6 provenait d'un unique chromosome paternel, et qui ne présentait aucun signe de diabète. L'analyse moléculaire plus détaillée de la lésion causale, si elle montre une microdélétion ou un réarrangement, permettra peut-être d'obtenir une réponse à ces questions.

[1. Abramowicz MA, et al. J Clin Invest 1994 ; 94: 418-21.]

[2. Sarvetnick N, et al. Cell 1988 ; 52 : 773-82.]

[3. Welch TR, et al. J Clin Invest $1990 ; 86: 675-8$.]

\section{- Structure tridimensionnelle du principal site neutralisant du} VIH. Le principal déterminant neutralisant de la protéine d'enveloppe (gp120) du VIH se trouve dans la boucle V3. Les anticorps neutralisants dirigés contre cette boucle bloquent l'entrée du virus dans les cellules et la formation de syncytia. Une courte séquence conservée de la boucle V3 est bordée par des séquences hypervariables. Les anticorps qui reconnaissent la région conservée ont une activité neutralisante large alors que les anticorps dirigés contre les régions variables ne neutralisent que certaines souches virales. En attendant que la structure de gpl20 soit élucidée, une équipe américaine a pu cristalliser un peptide de la boucle V3 associé à des fragments Fab d'anticorps neutralisants. Lorsqu'un peptide V3, contenant la région conservée, est associé au Fab d'un anticorps neutralisant à large spectre, les régions variables du peptide ne sont pas impliquées et l'interaction peut avoir lieu quelle que soit l'origine virale du peptide. Cette structure peptide-Fab est comparée à celle obtenue avec des anticorps neutralisants spécifiques d'isolats et reconnaissant un épitope chevauchant. Les acides aminés communs aux différents complexes prennent une conformation similaire, vraisemblablement très proche de celle qu'ils adoptent au sein de gp120. La résolution en trois dimensions du principal site de neutralisation pourrait donc être utilisée pour la mise au point de vaccins synthétiques ou d'inhibiteurs de fusion.

[Ghiara JB, et al. Science 1994 ; 264 82-9.] 
口 Synthèse d'immunoglobulines autoréactives chez les souris sans récepteur d'antigène. Dans un environnement dépourvu d'agents pathogènes, les souris sans aucun récepteur $\alpha \beta$ d'antigène $(\mathrm{TCR} \alpha /$;, due à l'absence du gène TCR $\alpha$ ) possèdent un grand nombre de cellules $\mathrm{B} \operatorname{IgM}^{+}, \operatorname{IgD}{ }^{+}$. Lorsqu'elles sont maintenues dans les conditions habituelles, la population cellulaire splénique $B$ et les concentrations d'IgE et d'IgGl sont plus importantes chez ces souris que chez les hétérozygotes $\mathrm{TCR} \alpha^{+} /$. Cela montre que, malgré l'absence de cellules $T \alpha \beta^{+}$, certains lymphocytes $B$ peuvent se différencier et sécréter ces isotypes d'Ig qui, normalement, sont sous la dépendance des lymphocytes $T$, ce qui soulève le problème du contrôle de la différenciation $B$ et de la mise en place de la tolérance du répertoire $B$ chez ces souris [1]. Selon des tests bien établis permettant d'appréhender le niveau d'autoréactivité, il semble que l'absence du TCR $\alpha \beta$ prédispose les souris à une expansion des cellules $\mathrm{B}$, à la sécrétion des isotypes « $\mathrm{T}$ dépendants " et à une auto-immunité généralisée. L'IL4, la principale interleukine responsable de la sécrétion d'IgGl et d'IgE, est produite par les cellules $T \alpha \beta^{+}$. Il doit donc exister, chez les souris TCR $\alpha /$, une source alternative de production d'IL4 qui pourrait être les lymphocytes $\mathrm{T} \gamma \delta[2]$. Ces lymphocytes pourraient fournir l'aide nécessaire aux lymphocytes B pour se différencier sans passer par la voie classique dépendante de CD40-gp39, utilisée par les cellules T $\alpha \beta C D 4$. Une production exacerbée d'IL4 par des lymphocytes $\mathrm{T} \gamma \delta$, ou par des cellules Th2 allogéniques dans le cas d'une réaction du greffon contre l'hôte, conduirait à une sécrétion d'autoanticorps. La production accrue d'IL4, dans ce cas comme chez les souris TCR $\alpha \%$, pourrait être liée, directement ou indirectement, à l'absence de cellules T $\alpha \beta C D 8^{+}$. Le déficit en cellules TCD8 pourrait contribuer à l'hyperplasie des lymphocytes B et à l'auto- immunité généralisée rencontrée dans les lymphopénies d'origine virale, comme le SIDA. Chez les sujets infectés par le VIH, comme chez les souris TCR $\alpha /$; le nombre de lymphocytes $\mathrm{T} \gamma \delta$ est souvent anormalement élevé [3].

[1. Wen L, et al. Nature $1994 ; 369$ : 654-8.]

[2. Banchereau J. médecine/sciences $1990 ; 6$ : 946-53.]

[3. De Paoli P, et al. Clin Exp Immunol $1991 ; 83$ : 187-91.] la moelle osseuse dans la présentation des antigènes tumoraux. Malgré la présence d'antigènes tumoraux reconnus in vitro par les lymphocytes $\mathrm{T}$, la croissance des tumeurs in vivo montre que ces lymphocytes $\mathrm{T}$ ne sont pas convenablement activés. Les lymphocytes cytotoxiques CD8 reconnaissent des antigènes qui dérivent de la dégradation de protéines endogènes, présentés par les molécules du complexe majeur d'histocompatibilité de classe I (CMH-I). C'est donc la tumeur elle-même qui devrait présenter ces antigènes endogènes pour activer les cellules T CD8 cytotoxiques. On sait pourtant que cela n'est pas forcément le cas ; l'expression du CMH-I par la tumeur n'est pas nécessaire à l'activation des lymphocytes T CD8 spécifiques des antigènes tumoraux, ce qui suggère que ceux-ci sont transférés à d'autres cellules $\mathrm{CMH}^{+}{ }^{+}$. Il est possible d'évaluer le rôle relatif des cellules tumorales et des cellules saines dans le déclenchement d'une réponse cytotoxique spécifique d'un antigène tumoral : les expériences de reconstitution de moelle osseuse chez la souris confirment cette observation en montrant que la réponse immunitaire dirigée contre un antigène de carcinome de côlon ne se met en place que si l'antigène est transféré aux cellules de la moelle osseuse pour être présenté aux lymphocytes T CD8 [1].
Le mécanisme par lequel des antigènes exogènes peuvent entrer efficacement dans le compartiment de classe I n'est pas clair, mais ces résultats suggèrent que la barrière dressée par le polymorphisme du $\mathrm{CMH}$ devant les stratégies vaccinales anticancer pourrait s'effondrer si les antigènes des cellules tumorales injectées étaient présentés par le $\mathrm{CMH}$ du receveur. La stratégie vaccinale antitumorale allogénique trouverait là sa justification, d'autant plus que des résultats récents suggèrent que les effets allogéniques pourraient stimuler les réponses immunes antitumorales [2].

[1. Huang AYC, et al. Science 1994 ; $264: 961-5$.]

[2. Plautz GE, et al. Proc Natl Acad Sci USA 1993 ; 90 : 4645-9.]

Parthénogenèse chez les souris déficientes en Mos. L'oncogène mos code pour une sérine-thréonine kinase. L'équivalent cellulaire $c$-mos n'est actif que dans les cellules germinales en cours de méiose. Il a été démontré que la protéine Mos était un composant essentiel du facteur cytostatique CSF (cytostatic factor) qui semble être responsable du blocage de la maturation des ovocytes en métaphase $2\left(\mathrm{~m} / \mathrm{s} n^{\circ} 2\right.$, vol. $6, p$. 162). La maturation reprend lors de la fécondation, marquée par l'expulsion du deuxième globule polaire, signe de l'achèvement de la seconde division méiotique. Par ailleurs, Mos est également indispensable, chez les amphibiens, dans les premiers stades de la maturation ovocytaire: rupture de la vésicule germinale, activation du MPF (maturation promoting factor), expulsion du premier globule polaire. Une équipe britannique de Cambridge [1] et une équipe japonaise [2] ont maintenant créé, par recombinaison homologue, des souris déficientes en Mos. Les animaux $\mathrm{mos}^{/ /}$se développent normalement et les mâles sont fer- 


\section{BRÈVES}

tiles. Cependant, la fertilité des femelles est très réduite. Cela est dû à une anomalie de l'arrêt de la maturation ovocytaire en métaphase 2 jusqu'à la fécondation par le spermatozoïde : la méiose se poursuit, le second globule polaire est expulsé et, parfois, les divisions débutent. Les ovaires de ces animaux forment des kystes et des tératomes ovariens. Chez les mammifères, par conséquent, Mos semble indispensable au blocage de la maturation au stade de la seconde division méiotique mais n'est pas requis pour les premières phases de la maturation ovocytaire ni pour la méiose des gamètes mâles.

[1. Colledge WH, et al. Nature 1994 ; 370 : 65-7.]

[2. Hashimoto $\mathrm{N}$, et al. Nature 1994 ; 370 : 68-71.]

Anaphylaxie en l'absence d'lgE. Les immunoglobulines E complexées à l'antigène se fixent à des récepteurs de forte affinité sur les mastocytes et les polynucléaires basophiles chez lesquels ils stimulent la libération de médiateurs du choc anaphylactique. Cependant, l'équipe de Philip Leder [1] vient de démontrer que des souris totalement déficientes en $\operatorname{IgE}$ à la suite d'une recombinaison homologue étaient tout aussi sensibles au choc anaphylactique que des souris normales. Ce choc passe pourtant bien par le système immunitaire puisqu'il n'est pas observé chez des souris immunodéprimées à la suite de l'inactivation des deux allèles du gène $R A G-2$ indispensable à la recombinaison des segments de gènes d'immunoglobulines et de récepteurs pour l'antigène des lymphocytes $T$ [1]. Il est probable que d'autres immunoglobulines que les $\mathrm{IgE}$ peuvent relayer la réponse anaphylactique à des antigènes.

[1. Oettgen HC, et al. Nature 1994 ; 370 : 367-70.]
Le récepteur nucléaire SF-1 : transcription et développement des surrénales et des gonades. Le développement des techniques d'inactivation génique par recombinaison homologue révèle de plus en plus d'exemples de la liaison entre le contrôle de la transcription et celui du développement et de la différenciation cellulaire. Ainsi, un facteur de transcription de la famille des récepteurs nucléaires, le facteur SF1, qui a d'abord été identifié par son rôle dans le contrôle de la transcription des gènes de plusieurs hydroxylases de la stéroïdogenèse, apparaît maintenant comme un régulateur essentiel du développement des tissus stéroïdogéniques, les surrénales et les gonades. De plus, ce même facteur semble jouer un rôle déterminant dans les mécanismes de la différenciation sexuelle. Chez les mammifères, le développement des testicules est déclenché par l'action du facteur de détermination testiculaire codé par le gène $S R Y$. Par la suite, les cellules de Sertoli sécrètent une hormone apparentée à la famille du facteur de croissance TGF, l'hormone antimüllérienne $(\mathrm{AMH})$, qui produit une régression des canaux müllériens [1]. Chez la femelle, en l'absence de AMH, les canaux müllériens se développent en oviductes, utérus et vagin. Bien que le gène $S R Y$ soit exprimé tôt au cours du développement, sa faible expression ultérieure ne rend pas compte des nombreux changements qui accompagnent l'organogenèse des gonades, tant chez les mâles que chez les femelles. Il semblait donc clair que d'autres facteurs devaient prendre le relais de $S R Y$ dans le contrôle de ces phénomènes de développement. Le travail récent des équipes de Keith L. Parker [1] (Durham, NC, USA) et Holly A. Ingraham [2] (San Francisco, CA, et Durham, NC, USA) a bien montré l'importance du facteur SF-1 dans le développement des organes stéroïdogéniques ainsi que dans la cascade de signaux nécessaires à la détermination sexuelle. Ainsi, dans un premier article, Luo et al. [3] ont montré que l'inactivation du gène $S F-1$ par recombinaison homologue entraîne une agénésie complète des surrénales et des gonades. Bien que les animaux homozygotes portant la mutation naissent dans les proportions attendues, tous meurent dans la semaine suivant la naissance. Il est vraisemblable qu'ils meurent d'insuffisance surrénalienne sévère puisqu'ils n'ont pas de surrénales. De plus, ces animaux n'ont pas de gonades et présentent un appareil génital interne femelle. Ce développement par défaut est observé chez tous les animaux dont le développement testiculaire est bloqué. Le travail de Shen et al. [4] révèle que l'action de $\mathrm{SF}-1$ sur la transcription du gène qui code pour l'AMH est vraisemblablement responsable du développement anormal des gonades. Ils ont montré, en effet, que le facteur SF-1 est un régulateur transcriptionnel important du gène $A M H$ par son action sur un site de liaison dans la région proximale du promoteur. En outre, il semble que l'action transcriptionnelle de SF-1 soit contrôlée par le domaine de liaison du ligand de ce récepteur nucléaire. Ainsi, de la même façon que l'activité d'autres facteurs de transcription de cette famille, tels les récepteurs des glucocorticoïdes, ceux des hormones sexuelles ou thyroïdes, il semble que SF-1 soit activé par un ligand qui reste à identifier mais dont la présence dans les gonades et les surrénales pourrait jouer un rôle clé dans le développement. Le facteur SF-1 est donc présent dans différentes cellules des surrénales et des gonades et son rôle va bien audelà de celui d'un facteur de transcription qui contrôle l'activité des gènes réalisant la stéroïdogenèse. Néanmoins, il est clair qu'en soi la présence de SF-1 ne suffit pas à rendre compte de toutes les fonctions des cellules l'exprimant, et que d'autres facteurs et d'autres marqueurs de la différenciation sexuelle doivent être impliqués dans ces phénomènes. Il ressort 
toutefois de ces études que les mécanismes de contrôle de la transcription et du développement utilisent un même régulateur, SF-1. Bien qu'elle ait été supposée depuis longtemps, la relation étroite qui existe entre transcription et développement nous apparaît de plus en plus évidente à mesure que l'étude des facteurs impliqués devient précise. Il n'y a pas de doute que les prochaines années nous réservent bien des découvertes sur d'autres régulateurs communs de la transcription et du développement.

[1. Jost A. médecine/sciences 1991; 7 : 263-75.]

[2. Josso $\mathrm{N}$, et al. médecine/sciences $1987 ; 3$ : 444-52.]

[3. Luo X, et al. Cell 1994; 77 : 48190.]

[4. Shen WH, et al. Cell 1994; 77 : 651-61.]

Une protéine cytosolique du virus Herpes simplex bloque la présentation des antigènes aux lymphocytes T CD8. Les lymphocytes T CD8 cytotoxiques sont les chefs de file de la lutte contre les virus mais dans quelques cas, pourtant, comme celui de la réponse contre le virus Herpes simplex (HSV), les lymphocytes $\mathrm{T}$ cytotoxiques antivirus expriment plus fréquemment CD4, habituellement porté par les lymphocytes $\mathrm{T}$ auxiliaires. Les lymphocytes $\mathrm{T}$ CD8 reconnaissent les peptides antigéniques liés aux molécules de classe I du complexe majeur d'histocompatibilité (CMHI) alors que les lymphocytes T CD4 sont stimulés par la reconnaissance des peptides liés au CMH-II. Au cours de la réponse cytotoxique anti-HSV, la disproportion en faveur des lymphocytes T CD4 et la résistance des fibroblastes humains, infectés par HSV, à l'activité cytotoxique des lymphocytes T CD8 spécifiques du virus indiquent que la présentation des antigènes par le CMH-I doit être altérée au cours de l'infection. En fait, HSV bloque le transport du CMH-I qui est retenu dans le (RE) et le cis-Golgi. Les infections avec des virus mutants montrent que l'expression de la protéine virale ICP47 est nécessaire et suffisante pour bloquer le transport intracellulaire du CMH-I et inhiber la lyse des fibroblastes infectés par les lymphocytes T CD8. ICP47 est une protéine cytosolique, non associée aux membranes, qui pourrait agir sur la production des peptides par les protéasomes ou sur leur translocation dans le réticulum endoplasmique. Comme l'adénovirus, HSV entre donc dans la catégorie des virus qui échappent à la détection par les lymphocytes $T$ CD8 en perturbant la voie de présentation des antigènes par le CMH-I.

[York IA, et al. Cell 1994; 77 : 52535.]

Localisation $d u$ gène de la sclérose latérale amyotrophique récessive sur le chromosome 2 . On sait que le gène de la forme autosomique dominante de la sclérose latérale amyotrophique familiale (ALSl) siège sur le chromosome 21 en 2 lq22. Une équipe américaine et tunisienne vient d'étudier la localisation de la forme récessive (ALS2), caractérisée par un début plus précoce et une évolution plus lente. Cette recherche a été effectuée sur une famille tunisienne étendue [1]. Le locus a été fixé sur le chromosome 2 en 2q33-q35, avec un lod score atteignant 8,2 .

[1. Hentati A, et al. Nature Genet $1994 ; 7$ : 425-8.] 